\title{
Conception of a C-Arm Parametrization Device Evaluated by an Inverse Registration Approach: An application for 2D/3D Registration in Minimally Invasive Surgery
}

\begin{abstract}
Camera resectioning is the process of finding the intrinsic and extrinsic parameters of the pinhole camera model. A mobile X-ray device (C-arm) can be represented with the pinhole camera model. The $\mathrm{C}$-arm parameters can be determined with a parametrization device and the direct linear transformation (DLT) algorithm. We present the conception process of a parametrization device and further optimizations of the DLT algorithm. The evaluation of the parametrization quality is carried out with an inverse registration approach on a testing device. The seven landmarks in the testing device are compared on an X-ray image and a digitally reconstructed radiograph (DRR). The average distance of the corresponding landmarks between imaging modalities is 2.5 pixels. This deviation indicates an adequate design of the parametrization device for a $2 \mathrm{D} / 3 \mathrm{D}$ registration in minimally invasive surgery.
\end{abstract}

Keywords: C-arm, parametrization device, camera resectioning, $2 \mathrm{D} / 3 \mathrm{D}$ registration.

\section{https://doi.org/10.1515/cdbme-2021-1006}

\section{Introduction}

In minimally invasive surgery, a patient's $3 \mathrm{D}$ pre-operative modality, such as a CT-Scan, is registered with the patient's position and orientation (pose) within the operating room (OR). A common approach is the $2 \mathrm{D} / 3 \mathrm{D}$ registration, which additionally uses an intra-operative modality as an X-ray

\footnotetext{
*Corresponding author: Julio C. Alvarez-Gomez: Institute of Control Engineering and Center for Sensor Systems (ZESS), University of Siegen, Siegen, Germany, E-mail: julio.agomez@uni-siegen.de. Hubert Roth and Jürgen Wahrburg: Institute of Control Engineering and Center for Sensor Systems (ZESS), University of Siegen, Siegen, Germany
}

image taken with a mobile X-ray device (C-arm.) The 2D/3D registration is calculated with a chain of transformation matrices, aiming to transform from the pre-operative modality to the patient pose. One of these transformations describes the $\mathrm{C}$-arm parameters, represented by the pinhole camera model. The $\mathrm{C}$-arm parameters can be found using the direct linear transformation (DLT) algorithm and a parametrization device. Due to the size and weight of the $\mathrm{C}$-arms, their structures are prone to bending. It is not trivial to find an analytical description to determine these deformations since they change as a function of the rotation and extension of the $\mathrm{C}$-arm with respect to its fixed body [1]. Those deformations in the structure affect the $\mathrm{C}$-arm parameters, i.e., they depend on the $\mathrm{C}$-arm pose. Some $\mathrm{C}$-arms with 3D reconstruction capability must know the $\mathrm{C}$-arm parameters in their range of motion. One approach is to build a look-up table of the $\mathrm{C}$-arm parameters as a function of its pose [2]. As these look-up tables are not available to the $\mathrm{C}$-arm user, a separate parametrization must be done if the $\mathrm{C}$-arm parameters are needed. In this paper, the conception of a parametrization device is elaborated. The evaluation of the parametrization is executed with the DLT algorithm and evaluated using an inverse registration approach. Finally, an optimizer is run over the DLT algorithm to improve the $\mathrm{C}$-arm parametrization.

\section{Materials and Methods}

One modern tool used inside the $\mathrm{OR}$ is the surgical navigation system. This device returns the pose of rigid bodies (RB) with respect to the camera reference frame, $\{\mathrm{OTS}\}$. In our research on $2 \mathrm{D} / 3 \mathrm{D}$ registration in minimally invasive surgery, we use two RBs, one built into the C-arm parametrization device $\{C R B\}$ and one attached to the patient's anatomy $\{A R B\}$.

Some studies have shown that the pinhole camera model is a good descriptor of a C-arm [3]. The pinhole camera model represents a camera as a focal point (optical center) and a 
retinal plane, where the images are rendered. The camera parameters are divided into intrinsic and extrinsic. The intrinsic parameters describe the optical arrangement of the camera. The extrinsic parameters compose the transformation from the camera reference frame $\{\mathrm{C}-\mathrm{ARM}\}$, located on the optical center, to an external reference frame $\{C R B\}$, i.e., ${ }^{C R B} \boldsymbol{T}_{C-A R M}$.

The intrinsic and extrinsic camera parameters can be found with a set of steel beads (fiducials) and the DLT algorithm. The coordinates of the fiducials in space and their corresponding projections on the X-ray image must be known. As a requirement of the DLT algorithm, the set of fiducials must be greater than six with no more than four coplanar as some rows of the linear equation system would become linearly dependent [4]. The literature states that an optimal result is achieved with more than 35 fiducials [5]. However, many fiducials make an impractical solution for the $2 \mathrm{D} / 3 \mathrm{D}$ registration since many fiducials substantially obstruct the body segment in the X-ray image. Some simulations were performed to determine the number of fiducials that make the DLT algorithm calculate accurate results. We created sizes of fiducials in a size range of 6 to 15, randomly created the camera parameters, and calculated the $2 \mathrm{D}$ projection of the fiducials. Gaussian noise is added to the coordinates of the fiducials. With the $3 \mathrm{D}$ and $2 \mathrm{D}$ sets with noise, the camera parameters are computed and compared to the actual parameters. A set of 12 or more fiducials calculates the intrinsic and extrinsic parameters with $0.53 \%$ relative error.

A parametrization device containing 12 fiducials is designed. Further simulations are run using a digitally reconstructed radiograph (DRR) module. The DRR uses a 3D volume as input and the transformation of the volume $\{D I C O M\}$ to the DRR reference frame $\{D R R\}$. The DRR renders an image using perspective projection from a point ray source. This working principle is consistent with the pinhole camera model. Therefore, there is correspondence between the DRR and the pinhole camera parameters. A set of 1000 DRR images with different camera parameters and DRR poses are

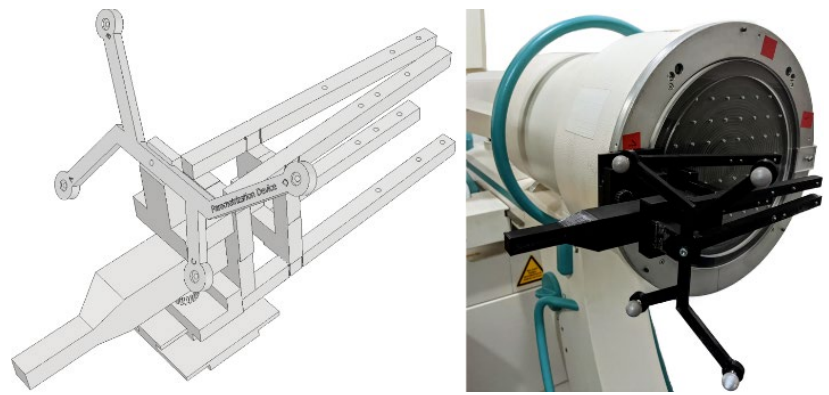

Figure 1: CAD design of the parametrization device on the left, installed device on the right created. The coordinates of the fiducials in $3 \mathrm{D}$ are known, and the $2 \mathrm{D}$ coordinates are detected from the DRR images. The evaluation is performed by calculating the $\mathrm{C}$-arm parameters and comparing them with the parameters used to generate the DRR image. The experiment shows that the $\mathrm{C}$-arm parametrization using the parametrization device is calculated with a relative error of $0.682 \%$. This error shows promising results to advance with the construction of the parametrization device.

The parametrization device is designed complying the DLT requirements for the fiducials, and the positions of the fiducials are maintained by a built-in RB, $\{\mathrm{CRB}\}$ (See Figure 1). It means that this $R B$ is the external frame used for the extrinsic parameters. We use the navigation system and a spherical hollow-tip tracking pointer to calculate the position of the fiducials once the parametrization device is built. It is necessary to find the $3 \mathrm{D}$ coordinates after the fabrication of the parameterization device due to the inaccuracies added when using a $3 \mathrm{D}$ printer.

As reported in the literature, the structures of the C-arms are prone to bending, creating fluctuations in the intrinsic parameters depending on the $\mathrm{C}$-arm pose [1]. The used C-arm, a Ziehm Vario 3D, offers the possibility to make scans. One scan takes $130 \mathrm{X}$-ray images along the $\mathrm{C}$-arm arc, which has a $135^{\circ}$ stroke. Ten of these scans are performed, varying the rotation point of the $\mathrm{C}$-arm, thus creating 1300 unique images. For each of the images, the intrinsic and extrinsic parameters are calculated.

One of the simplest parameters to analyze is the focal length since the $\mathrm{C}$-arm bending is expressed as a one-dimensional parameter. The parameters of the ten scans are ordered according to the angle of the $\mathrm{C}$-arm arc. The scans start at $90^{\circ}$ from the lateral (LAT) projection, rotating around $0^{\circ}$, the anterior-posterior (AP) projection, and ending at $-35^{\circ}$. The values are further gathered in groups of $10^{\circ}$ range. For each range, there are 100 samples, which are averaged. The plot of focal length vs. angle of the $\mathrm{C}$-arm can be seen in Figure 2. It can be noticed how the bending of the $\mathrm{C}$-arm

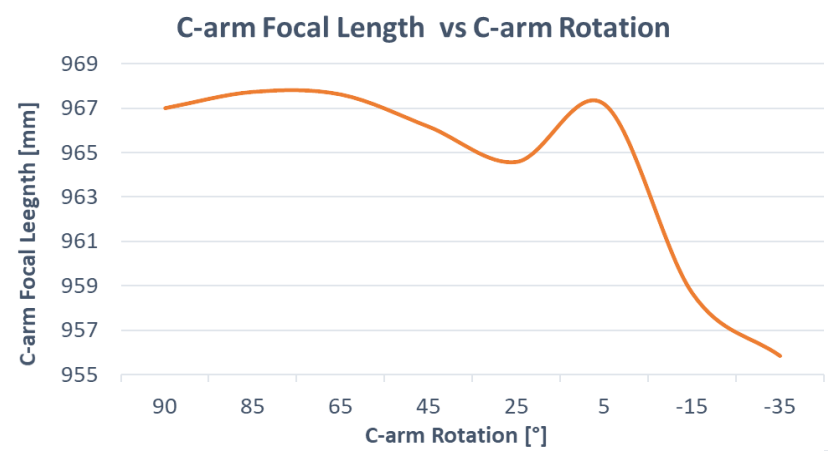

Figure 2: Focal length vs angle of the C-arm 
structure affects its focal length. From the values at $90^{\circ}$ (LAT projection) and $0^{\circ}$ (AP projection), the focal length is computed to be $967.17 \mathrm{~mm}$ and $966.99 \mathrm{~mm}$, respectively.

In the $2 \mathrm{D} / 3 \mathrm{D}$ registration, the patient's DICOM is used in the DRR module to find the $\{\mathrm{DICOM}\}$ to $\{\mathrm{DRR}\}$ pose, ${ }^{D R R} \boldsymbol{T}_{\text {DICOM }}$. The registration is achieved when the most similar DRR image is found compared to an X-ray image taken with the $\mathrm{C}$-arm in the $\mathrm{OR}$. The registration result is the transformation $\{\mathrm{DICOM}\}$ to the $\mathrm{RB}$ fixed to the patient, $\{\mathrm{ARB}\}$. (see eq. 1).

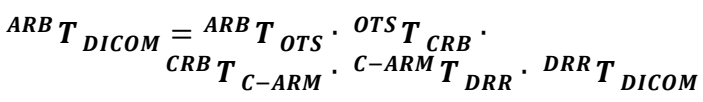

To ascertain the accuracy of the found $\mathrm{C}$-arm parameters, let a testing device be introduced (See Figure 3) with the following characteristics:

- $\quad$ A CT-scan of the device is available as a DICOM image.

- The device has seven landmarks that can be detected physically and in the available imaging modalities.

- The device has an RB rigidly attached, which keeps its registration, i.e., ${ }^{A R B} \boldsymbol{T}_{\text {DICOM }}$.

- This ${ }^{A R B} \boldsymbol{T}_{\text {DICOM }}$ has an error smaller than $1 \mathrm{~mm}$.

From the transformation chain of the $2 \mathrm{D} / 3 \mathrm{D}$ registration (see eq. 1), the transformation $\{\mathrm{DICOM}\}$ to $\{\mathrm{ARB}\}$ is known. Solving Equation 1 for ${ }^{D R R} \boldsymbol{T}_{\text {DICOM }}$ allows us to render a DRR image from the same perspective as the $\mathrm{X}$-ray image used for the $\mathrm{C}$-arm parametrization. The device and $\mathrm{C}$-arm poses are taken with the navigation system, ${ }^{\text {OTS }} \boldsymbol{T}_{A R B}$ and ${ }^{O T S} \boldsymbol{T}_{C R B}$, respectively. We compare the X-ray image of the testing device and the DRR image created by the C-arm parameters, i.e., ${ }^{C R B} \boldsymbol{T}_{C-A R M}$. If the $\mathrm{C}$-arm parameters are perfectly calculated, the corresponding coordinates of the landmarks in the DRR and the X-ray image would be located at the same

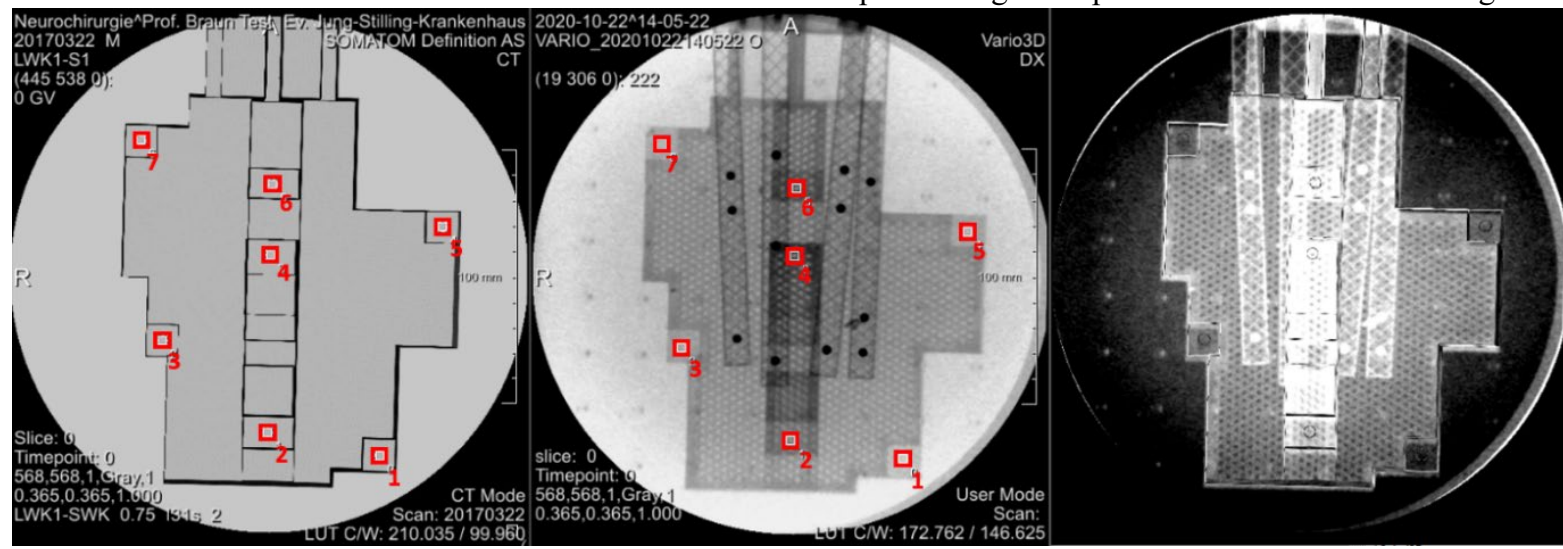

Figure 4: On the left image, the DRR image with the detected landmarks. On the center, the X-ray image with the detected landmarks. A subtraction operation of the DRR image and the X-ray image is visible on the right

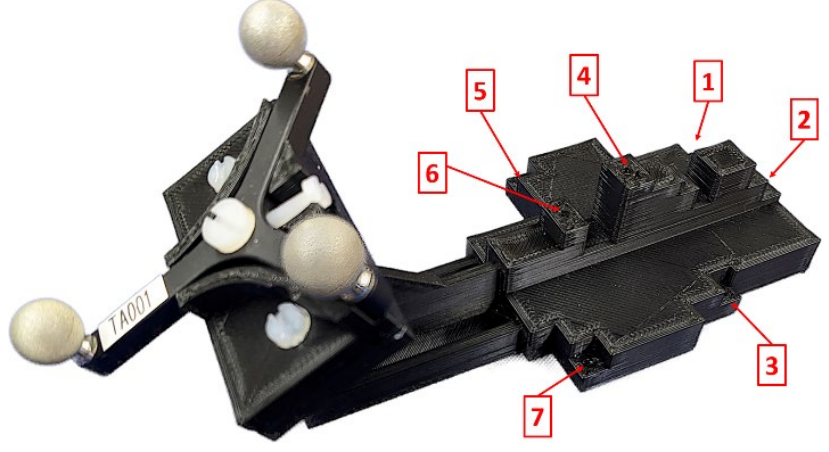

Figure 3: Device to test the C-arm calibration

point. The Euclidian distance of each pair of corresponding landmarks is used, meaning seven Euclidian distances are calculated per trial. The divergence between landmarks gives us the quality of the $\mathrm{C}$-arm parametrization. We called this evaluation process: inverse registration approach.

\section{Results}

Five AP and five LAT X-ray images of the testing device are taken using the $\mathrm{C}$-arm while taken measurements with the optical tracking system. After running the DLT algorithm, the average distance between the corresponding landmarks is about 35.7 pixels. This mismatch between X-ray and DRR images indicates inaccuracies in the $\mathrm{C}$-arm parametrization. The averaged focal length values of the $\operatorname{AP}\left(0^{\circ}\right)$ and LAT $\left(90^{\circ}\right)$ projections taken from Figure 2 are used as constraints to optimize the coordinates of the fiducials during the calculation of the C-arm parameters with the DLT algorithm [6]. The implemented optimizer is a simplex hill, and the delta steps are changes of $0.02 \mathrm{~mm}$ in the coordinates of the fiducials. After implementing this optimization over the DLT Algorithm, the 
results of the last experiment are recalculated. The average distance is $2.5 \mathrm{px}$, compared with $35.7 \mathrm{px}$ when the parameters are calculated without optimization.

The results indicate that the current approach finds a $\mathrm{C}$-arm camera matrix that models the real device with a difference of $2.5 \mathrm{px}$ on average. The graphical result for one of the AP images is illustrated in Figure 4. Since the pixel spacing of the Ziehm Vario 3D is $0.345 \mathrm{~mm} / \mathrm{px}$, the average distance error is $0.8625 \mathrm{~mm}$. This result is considered adequate for the $\mathrm{C}$-arm parametrization. Therefore, each X-ray image used in our registration process uses the proposed device and procedure to find the $\mathrm{C}$-arm parameters.

\section{Discussion and Conclusions}

In our research in $2 \mathrm{D} / 3 \mathrm{D}$ registration in minimally invasive surgery, the transformation of the pre-operative data to the patient in the operating room (OR) is found by taken X-ray images from a mobile X-ray device (C-arm) inside the OR. The $\mathrm{C}$-arm parameters are one of the transformations in the registration equation (see eq. 1). Since the $\mathrm{C}$-arm images are formed from an ideal focal X-ray source, the $\mathrm{C}$-arm can be mathematically represented using the pinhole camera model. With this approach, the intrinsic and extrinsic parameters of the $\mathrm{C}$-arm can be known. The intrinsic parameters refer to the $\mathrm{C}$-arm optical arrangement, which is theoretically static, but it is pose-dependent in practice. The extrinsic parameters represent the transformation from the $\mathrm{C}$-arm reference frame $\{\mathrm{C}-\mathrm{ARM}\}$ to the external frame describing the positions of the fiducials $\{\mathrm{CRB}\}$.

The direct linear transformation (DLT) algorithm is used to find the camera parameters. The accuracy of the results depends on the number of used points (fiducials), which must be greater than six. A trade-off must be found between the number of used fiducials and the accuracy since the more fiducials there are, the more the body segment is obstructed. Twelve fiducials have been found to provide sufficient accuracy and keep the image obstruction to a minimum.

A set of scans is executed covering the entire range of motion of the $\mathrm{C}$-arm. The results of the scans are used to plot the curve focal length vs. C-arm angle, showing a volatile focal length throughout the range of motion. Presumably, the $\mathrm{C}$-arm bending is the cause of the focal length variation.

A test for the $\mathrm{C}$-arm parametrization is carried out using a testing device with seven landmarks and an inverse registration approach. The test result provides a pose for the digitally reconstructed radiograph (DRR) module, which creates a DRR image from the DICOM of the testing device.
Ideal $\mathrm{C}$-arm parameters make a perfect match between the DRR and the X-ray image. The error in estimating the parameters is measured as a function of the Euclidian distance between corresponding landmarks in the DRR and X-ray image. The initial results give an average distance among corresponding landmarks of $35.7 \mathrm{px}$. Later, an optimizer is implemented to improve the $\mathrm{C}$-arm parametrization. In this final stage, the average distance of corresponded landmarks between DRR images created with the found camera parameters and the actual X-ray images is reduced from $35.7 \mathrm{px}$ to $2.5 \mathrm{px}$. This disparity between landmarks is considered adequate for the $\mathrm{C}$-arm parametrization. Therefore, each X-ray image in our 2D/3D registration approach for minimally invasive surgery includes the parameterization device, which finds the $\mathrm{C}$-arm parameters using the proposed parameterization process.

\section{Author Statement}

Research funding: Part of this work is funded by the German Federal Ministry of Education and Research (KMU-innovativ: contract number 13GW0175B). Conflict of interest: Authors state no conflict of interest. Informed consent: Informed consent has been obtained from all individuals included in this study. Ethical approval: The research related to human use complies with all the relevant national regulations, institutional policies and was performed in accordance with the tenets of the Helsinki Declaration, and has been approved by the authors' institutional review board or equivalent committee.

\section{References}

[1] Abbas T. Correction of Distortion Aberration in Electron Magnetic Lenses. International Journal of Applied Physics 2016;2;1-5.

[2] Schumann S, Dong X, Puls M, Nolte L, Zheng G. Calibration Of C-Arm for Orthopedic Interventions Via Statistical ModelBased Distortion Correction and Robust Phantom Detection. IEEE International Symposium on Biomedical Imaging 2012;1204-1207.

[3] Yaniv Z, Joskowicz L, Simkin A, Garza-Jinich M, Milgrom C. Fluroscopic Image Processing for Computer-Aided Ortohpaedic Surgery. International Conference on Medical Image Computing and Computer-Assisted Intervention.1998;1496;325-334,

[4] Faugeras O. Three-Dimensional Computer Vision A Geometrical Viewpoint. Cambridge: The MIT Press 1993.

[5] Hartley R, Zisserman A. Multiple View Geometry in Computer Vision, 2nd Ed. New York: Cambridge University Press 2004.

[6] Mennessier C, Spencer B, Clackdoyle R, Conneau A, Xu T. Distortion Correction, Geometric Calibration, and Volume Reconstruction for an Isocentric C-Arm X-Ray System. IEEE Nuclear Science Symposium Conference Record 2011;29432947. 